\title{
La utilización de las encuestas de movilidad domiciliaria para un análisis de accesibilidad Aplicación para el caso de acceso a Centros Educativos en el Área Metropolitana de Buenos Aires
}

Natalia Verónica Neri

Instituto de Transporte, Universidad Nacional de San Martín, Argentina. https://orcid.org/oooo-0002-9678-1328

\section{Carla Galeota}

Instituto de Transporte, Universidad Nacional de San Martín, Argentina. https://orcid.org/oooo-0003-4041-7399

\section{Lucila Capelli}

Instituto de Transporte, Universidad Nacional de San Martín, Argentina. https://orcid.org/oooo-0002-2647-6918

Recibido: 4 de junio de 2019. Aceptado: 27 de abril de 2020.

\begin{abstract}
Resumen
Entender los desafíos de accesibilidad es una demanda creciente para las políticas públicas de transporte y movilidad del Área Metropolitana de Buenos Aires. Este trabajo describe la metodología utilizada para analizar la accesibilidad a centros educativos de niños, niñas y adolescentes (NNA) en el Área Metropolitana de Buenos Aires a partir de una encuesta de movilidad domiciliaria. De esta manera el artículo aborda cómo una encuesta domiciliaria puede utilizarse para un análisis territorial, dejando manifiesto sus debilidades y fortalezas como herramienta metodológica.
\end{abstract}

Palabras clave: Movilidad. Territorio. Encuesta. Accesibilidad. Niños, niñas, adolescentes.

Analysis of accessibility to educational centers from mobility surveys: application for the case of Metropolitan Area of Buenos Aires

\footnotetext{
Abstract

Understanding accessibility challenges is a growing demand for public transport and mobility policies in the Region. This paper describes the methodology used to analyze the accessibility to educational centers for children and adolescents (NNA, in spanish)
} 
in the Metropolitan Area of Buenos Aires, based on a home mobility survey. In this way, the article proposes to discuss how a household survey can be used for a territorial analysis, revealing its weaknesses and strengths as a methodological tool.

Palavras-chave: Mobilidade. Território. Survey. Acessibilidade. Meninos, meninas, adolescentes Keywords: Mobility. Territory. Survey. Accessibility. Boys, girls, teenagers.

\section{Introduccion}

El Área Metropolitana de Buenos Aires (AMBA) suele ser en materia de estudios de transporte un área rica en datos estadísticos de movilidad. A partir de financiamiento externo se ha desarrollado durante los años 2009 y 2010 una encuesta probabilística representativa de la movilidad en la cual se ha podido avanzar en distintos ámbitos profesionales y académicos con el estudio de características de la movilidad de las personas que residen en esta área.

El presente artículo se basa en una investigación realizada en el marco de un Proyecto de Desarrollo Tecnológico y Social (PDTS) del Ministerio de Ciencia, Tecnología e Innovación Productiva (MINCYT), realizado por el Instituto del Transporte, en conjunto con la Escuela de Humanidades de la Universidad Nacional de San Martin y el Instituto Gioja de la Universidad Nacional de Buenos Aires. El proyecto culminó con la publicación de un artículo denominado "La movilidad y las condiciones de accesibilidad a centros educativos en el Área Metropolitana de Buenos Aires" (Bertranou, et al., 2020).

Con base en este trabajo, se abordó la accesibilidad en el AMBA a centros educativos, utilizando como fuente de información la más representativa hasta el momento para el Área Metropolitana de Buenos Aires: la Encuesta de Movilidad Domiciliaria (ENMODO).

En este artículo se describe la metodología de análisis y abordaje de la problemática de la accesibilidad al transporte público, a partir de la territorialización de la encuesta ENMODO para el grupo poblacional de los niños, niñas y adolescentes entre 3 y 17 años (NNA) a centros educativos en el AMBA, resaltando las fortalezas y debilidades para un apropiado análisis territorial de la accesibilidad: desde el diseño de la encuesta, la forma de presentación de los resultados, y la representatividad de los mismos cuando se representa la misma en una escala territorial.

\section{Sobre la encuesta}

La ENMODO, realizada por la entonces Secretaría de Transporte de la Nación, fue diseñada para realizar una caracterización de los patrones la movilidad del AMBA, tanto cualitativa como cuantitativamente para entender la problemática de movilidad de la población de la ciudad primada de la Argentina. La encuesta fue realizada durante fines del año 2009 y principios del 2010, con un relevamiento final de 22.500 hogares (representando aproximadamente 80.000 personas), registrando todos los viajes de los integrantes al día anterior a la encuesta, con sus respectivas "etapas de viaje".

Esta encuesta ha permitido entender la movilidad metropolitana, logrando la desagregación de datos por (entre otros): sexo, nivel socio económico, edad, condición ante la movilidad (por ejemplo, si poseen o no automóvil particular), estructura de 
la familia, motivos de los viajes, frecuencia. A su vez, la encuesta captó la movilidad y accesibilidad para diferentes motivos de viaje, como ser: trabajo, educación, salud, entretenimiento, deportes, entre otros; y permitió asociar esta información a distintos grupos de población de acuerdo con características socioeconómicas o de localización espacial (territorialización). Así, el diseño de la muestra fue realizado con un dominio de estimación único, el AMBA, por lo tanto, algunas variables de análisis no pudieron ser desagregadas para dominios menores. En la Figura 1 puede observarse la ubicación geográfica de las encuentras, evidenciando que la misma no ha incluido la totalidad de los partidos del AMBA. ${ }^{1}$

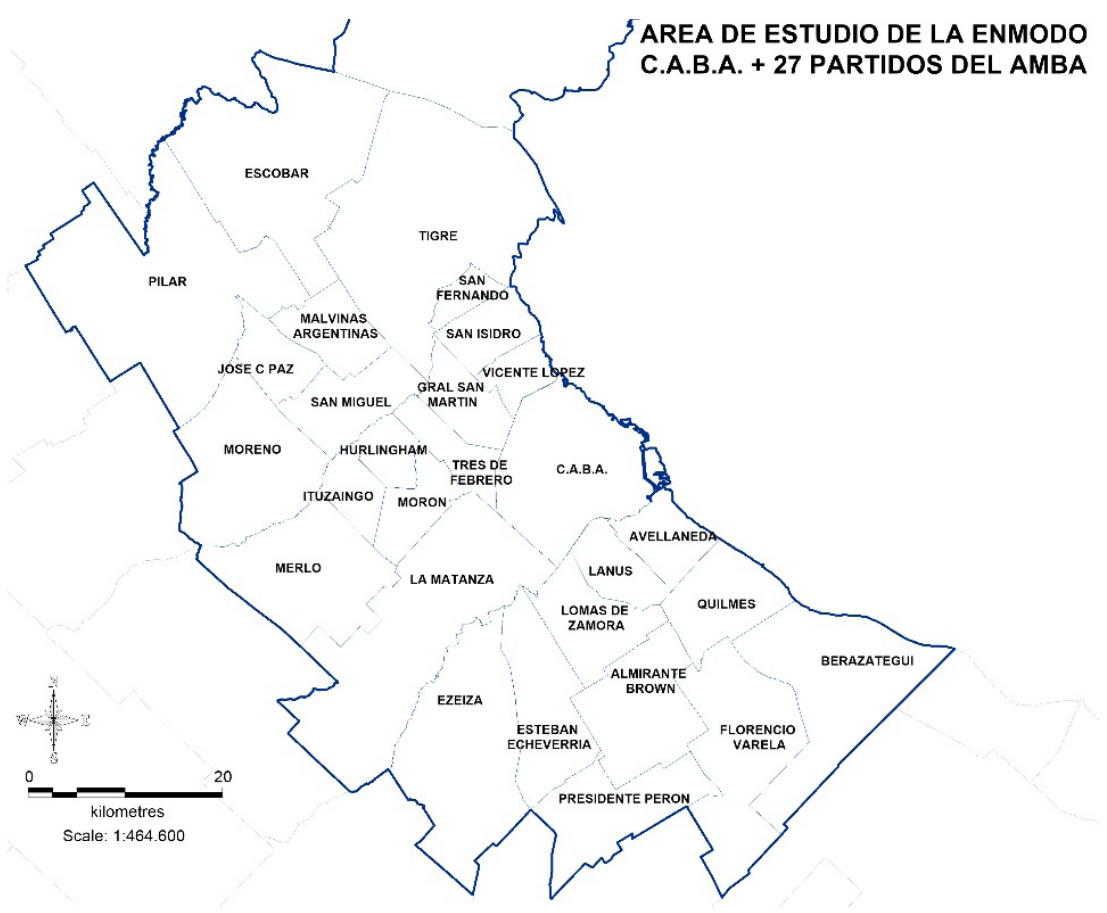

Figura 1. Distribución territorial de las encuestas. Fuente: elaboración propia en base a ENMODO (2010).

\section{Territorializacion y metodologia utilizada}

Si bien la ENMODO ha resultado ser representativa a nivel total población, utilizando la unidad de análisis a partir del radio censal (mínima unidad de análisis geográfica con base en los Censos Nacionales de Población), al desagregar los datos para analizar diferentes variables, no resulta representativa para para cada una de las variables en particular necesariamente.

En este sentido, con el objetivo de medir la accesibilidad a los centros educativos por parte de los niños, niñas y adolescentes (en adelante, NNA), se realizó un primer abordaje a nivel municipal, encontrando que los datos no son representativos con este nivel

1 Cabe destacar, que las encuestas no se realizaron en los 44 partidos que integran el AMBA, sino que el área definida por la ENMODO contempló la Ciudad Autónoma de Buenos Aires y 27 partidos de la Provincia de Buenos Aires, a saber: Vicente López, San Isidro, General San Martín, Tres de Febrero, Hurlingham, Ituzaingó, Morón, La Matanza, Lomas de Zamora, Lanús, Avellaneda, Quilmes, Almirante Brown, Esteban Echeverría, Ezeiza, Merlo, Moreno, San Miguel, José C. Paz, Malvinas Argentinas, San Fernando, Tigre, Escobar, Pilar, Presidente Perón, Florencio Varela y Berazategui. 
de desagregación: por el motivo del viaje (estudio) y por la población objetivo (NNA). Por este motivo, se modificó el corte territorial en función de la muestra, resultando en una agregación de municipios por zonas geográficas: CABA (dividida en CABA norte y CABA sur), Corona 1 (dividida en Norte, Sur, Oeste) y Corona 2 (dividida en Norte, Sur y Oeste). Es de destacar, que se han excluido los datos de Corona 3, por ser parciales (solo incluye los partidos de Escobar y Pilar - Norte) y además no resultó representativa.

Por último, es de destacar que, para procesar consultas en la base de datos, se ha utilizado el programa estadístico informático SPSS, el cual permitió realizar diferentes procesamientos dando como resultado tablas, que son las que se utilizaron para concluir en los diferentes cuadros del presente documento.

\section{Caracterizacion de los viajes}

Para conocer cómo se realizan los viajes a los centros educativos por los NNA, se analizaron las siguientes variables de movilidad:

》 Modo de transporte: Motorizado público, motorizado privado, no motorizado;

» Tiempo de Viaje;

》Distancia caminada para acceder al transporte público;

» Tiempo de espera del transporte público.

A partir de estas cuatro variables se analizaron las características de accesibilidad a centros educativos destacando las particularidades de los NNA. Para ello se conformaron tres grupos de NNA en relación con el nivel de educación al que pertenecen y las propias características etarias que influyen directamente sobre los patrones de viajes. El primer grupo son los niños y niñas de 3 a 5 años quienes asisten a centros de primera infancia, el segundo grupo son los niños y niñas de 6 a 12 años quienes asisten a centros de educación primaria (NNA 6 a 12 años), y por último el grupo de adolescentes de 13 a 17 años, quienes asisten generalmente a la educación de nivel secundario (NNA 13 a 17 años).

Por otra parte, se analizaron los viajes escolares que se realizan con un adulto acompañante, dado que el formulario de la encuesta ENMODO contempla un motivo específico que es "acompañando a centro educativo".

\section{Resultados de los procesamientos \\ Modo de Transporte}

El modo de transporte más utilizado para asistir a centros educativos por parte de los NNA es el "no motorizado". Ahora bien, entre aquella proporción que utiliza un modo de transporte "motorizado", el análisis desagregado de los datos por grupo de NNA y zona geográfica demuestra una variación en cuanto a lo empleado por cada uno de estos: en los NNA de 3 a 5 años existe una mayor proporción de viajes en transporte privado, mientras que en los NNA de 13 a 17 años los viajes se realizan casi en su totalidad en transporte público. 
Cuadro 1. Modo de Transporte según grupo de NNA y localización. Fuente: Elaboración propia, en base a Encuesta de Movilidad en el Área Metropolitana de Buenos Aires, ENMODO, 2009-2010.

\begin{tabular}{|c|c|c|c|c|}
\hline Zona Geográfica & Modo de Transporte & $\begin{array}{l}\text { NNA } 3 \text { a } 5 \\
\text { años }\end{array}$ & $\begin{array}{l}\text { NNA } 6 \text { a } 12 \\
\text { años }\end{array}$ & $\begin{array}{l}\text { NNA } 13 \text { a } \\
17 \text { años }\end{array}$ \\
\hline \multirow{3}{*}{ CABA NORTE } & Público & $13 \%$ & $18 \%$ & $42 \%$ \\
\hline & Privado & $39 \%$ & $30 \%$ & $14 \%$ \\
\hline & No Motorizado & $48 \%$ & $51 \%$ & $44 \%$ \\
\hline \multirow{3}{*}{ CABA SUR } & Público & $16 \%$ & $21 \%$ & $52 \%$ \\
\hline & Privado & $22 \%$ & $19 \%$ & $7 \%$ \\
\hline & No Motorizado & $62 \%$ & $60 \%$ & $42 \%$ \\
\hline \multirow{3}{*}{$\begin{array}{l}\text { CORONA } 1 \text { NORTE Y SUR } \\
\text { (promedio) }\end{array}$} & Público & $14 \%$ & $22 \%$ & $45 \%$ \\
\hline & Privado & $32 \%$ & $26 \%$ & $10 \%$ \\
\hline & No Motorizado & $54 \%$ & $52 \%$ & $44 \%$ \\
\hline \multirow{3}{*}{$\begin{array}{l}\text { RESTO CORONA } 1 \text { Y } 2 \\
\text { (promedio) }\end{array}$} & Público & $13 \%$ & $22 \%$ & $32 \%$ \\
\hline & Privado & $19 \%$ & $16 \%$ & $17 \%$ \\
\hline & No Motorizado & $67 \%$ & $62 \%$ & $52 \%$ \\
\hline \multirow{3}{*}{ TOTAL } & Público & $14 \%$ & $21 \%$ & $44 \%$ \\
\hline & Privado & $26 \%$ & $21 \%$ & $8 \%$ \\
\hline & No Motorizado & $60 \%$ & $58 \%$ & $48 \%$ \\
\hline
\end{tabular}

Es de destacar que el 60\% de los NNA de 3 a 5 años utilizan los modos de transporte no motorizados. A mayor desagregación se evidencian diferencias entre las zonas geográficas: el 67\% de la población que reside en la zona "Resto Corona 1 y 2", utiliza modos no motorizados, mientras que para la población de CABA Norte el número desciende al 48\% (zona que se esperaba una accesibilidad mayor en modos no motorizados).

Por otra parte, analizando los viajes en modos privados (el cual incluye automóvil particular, taxi, remis ${ }^{2}$, chárter ${ }^{3}$, transporte escolar ${ }^{4}$ y/o motocicleta) se destaca la diferencia según zona geográfica y edad. A nivel general, los viajes de los NNA de 3 a 5 años en modos privados son los que poseen mayor proporción en el total etario, entendiendo que esto puede deberse a las necesidades de desplazamiento hacia las instituciones de primera infancia. Ahora bien, en la desagregación geográfica se observan diferencias territoriales, en donde la utilización de modos de transporte privado en su mayoría desciende conforme se aleja del centro hacia el sur de la ciudad.

En el cuadro 2, puede observarse el reparto modal de los viajes por grupo de NNA y por zona geográfica desagregando en: transporte a pie, automóvil particular, bicicleta, colectivo, taxi o remis, subte, transporte escolar, ferrocarril, y otros. Aquí puede notarse la baja incidencia a nivel general del uso de la bicicleta en relación con el viaje a pie (ambos, transporte no motorizado). La excepcionalidad para destacar es que, si tomamos la totalidad de los viajes realizados en bicicleta, en la Corona 2 se da la mayor cantidad de estos, destacando que en el grupo de NNA de 3 a 5 años el porcentaje de estos viajes asciende al 9,6\% del total de los realizados en este tipo de transporte,

2 El remis es un tipo de transporte muy similar al taxi, utilizado generalmente en Argentina y Uruguay, en donde el usuario se contacta con una agencia que disponibiliza autos con chofer para realizar un trayecto de origen/destino, abonándose por kilómetro o tramo recorrido.

3 El chárter es un servicio de transporte de oferta libre, que consiste en combis con capacidades que varían de 6 a 45 asientos generalmente, con orígenes y destinos fijados por el operador. La tarifa esta desregulada y la proponen los operadores.

$4 \mathrm{Al}$ igual que los chárter, el servicio de "transporte escolar" es un servicio de transporte de oferta libre, pero que se caracteriza por ser exclusivamente para el traslado de asistentes a los centros educativos, con origen en los domicilios de los asistes y destino en la institución escolar. Se caracterizan por ser combis/buses de color naranja, y se encuentran identificados con el nombre de "transporte escolar". 
correspondiéndose con un fuerte descenso del uso del automóvil, modo que se utiliza mayormente en CABA Norte, principalmente para viajes de este grupo de NNA.

Cuadro 2. Tipo de Transporte según grupo de NNA y localización. Fuente: Elaboración propia, en base a Encuesta de Movilidad en el Área Metropolitana de Buenos Aires, ENMODO, 2009-2010.

\begin{tabular}{|c|c|c|c|c|c|}
\hline $\begin{array}{l}\text { GRUPO } \\
\text { ETARIO }\end{array}$ & Tipo de Transporte & $\begin{array}{c}\text { CABA } \\
\text { NORTE }\end{array}$ & CABA SUR & CORONA 1 & CORONA 2 \\
\hline \multirow{9}{*}{ NNA 3 a 5} & A pie & $46,1 \%$ & $60,3 \%$ & $53,1 \%$ & $58,6 \%$ \\
\hline & Automóvil & $28,2 \%$ & $12,9 \%$ & $19,9 \%$ & $9,9 \%$ \\
\hline & Bicicleta & $1,4 \%$ & $0,0 \%$ & $2,8 \%$ & $9,6 \%$ \\
\hline & Colectivo & $14,2 \%$ & $17,0 \%$ & $14,0 \%$ & $12,9 \%$ \\
\hline & Taxi o remis & $5,5 \%$ & $1,9 \%$ & $1,6 \%$ & $1,7 \%$ \\
\hline & Subte & $0,7 \%$ & $0,0 \%$ & $\mathrm{n} / \mathrm{c}$ & $\mathrm{n} / \mathrm{c}$ \\
\hline & Transporte escolar & $3,2 \%$ & $5,9 \%$ & $6,2 \%$ & $5,8 \%$ \\
\hline & Ferrocarril & $0,0 \%$ & ०\% & $0,7 \%$ & $0,4 \%$ \\
\hline & Otros & $0,7 \%$ & $2,0 \%$ & $0,7 \%$ & $1,1 \%$ \\
\hline \multirow{9}{*}{ NNA 6 a 12} & A pie & $51,4 \%$ & $59,7 \%$ & $53,2 \%$ & $58,7 \%$ \\
\hline & Automóvil & $18,2 \%$ & $11,2 \%$ & $13,7 \%$ & $6,7 \%$ \\
\hline & Bicicleta & $0,0 \%$ & $0,1 \%$ & $1,0 \%$ & $4,1 \%$ \\
\hline & Colectivo & $16,2 \%$ & $20,6 \%$ & $21,3 \%$ & $22,5 \%$ \\
\hline & Taxi o remis & $0,1 \%$ & $2,2 \%$ & $0,9 \%$ & $1,3 \%$ \\
\hline & Subte & $1,1 \%$ & $1,0 \%$ & $\mathrm{n} / \mathrm{c}$ & $\mathrm{n} / \mathrm{c}$ \\
\hline & Transporte escolar & $8,9 \%$ & $4,8 \%$ & $8,4 \%$ & $5,7 \%$ \\
\hline & Ferrocarril & $0,9 \%$ & $0,0 \%$ & $0,6 \%$ & $0,2 \%$ \\
\hline & Otros & $3,2 \%$ & $0,4 \%$ & 0,9 & $0,8 \%$ \\
\hline \multirow{9}{*}{ NNA 13 a 17} & A pie & $44,0 \%$ & $41,8 \%$ & $45,0 \%$ & $51,7 \%$ \\
\hline & Automóvil & $10,6 \%$ & $5,7 \%$ & $5,5 \%$ & $2,9 \%$ \\
\hline & Bicicleta & $0,2 \%$ & $0,0 \%$ & $0,6 \%$ & $1,2 \%$ \\
\hline & Colectivo & $37,6 \%$ & $47,0 \%$ & $43,9 \%$ & $39,4 \%$ \\
\hline & Taxi o remis & $0,5 \%$ & $0,5 \%$ & $0,7 \%$ & $1,0 \%$ \\
\hline & Subte & $3,7 \%$ & $4,6 \%$ & $\mathrm{n} / \mathrm{c}$ & $\mathrm{n} / \mathrm{c}$ \\
\hline & Transporte escolar & $1,1 \%$ & $0,4 \%$ & $2,4 \%$ & $0,7 \%$ \\
\hline & Ferrocarril & ०\% & $0,0 \%$ & $1,0 \%$ & $1,8 \%$ \\
\hline & Otros & $2,3 \%$ & $0,0 \%$ & $0,9 \%$ & $1,3 \%$ \\
\hline
\end{tabular}

\section{Tiempo de viaje}

El análisis de esta variable pudo desagregarse para identificar en forma separada la duración de los viajes en transporte público en relación con el resto de los modos, entendiendo que existe una diferencia sustancial entre los promedios de tiempos de viaje según las características de la oferta del servicio (como trazas de los recorridos y cantidad de paradas del servicio).

En relación con la duración del viaje, la encuesta se segmentó en diferentes rangos temporales, a saber: 15 minutos o menos, de 16 a 30 minutos, de 31 a 45 minutos, $y$ más de 45 minutos. El cuadro 3 permite ver los resultados para todos los NNA. Los resultados desagregados por subcategoría territorial, así como por grupo de NNA no fueron significativos estadísticamente y por lo tanto se descartaron; probablemente porque "el tiempo de viaje" esté relacionado mayormente con las distancias entre los orígenes y destinos de viaje y no con características de los grupos de análisis. 
Cuadro 3. Duración del viaje en transporte público respecto del resto de los modos. Fuente: Elaboración propia, en base a Encuesta de Movilidad en el Área Metropolitana de Buenos Aires, ENMODO, 2009-2010.

\begin{tabular}{|l|c|c|}
\hline Duración del viaje promedio NNA & Todos los modos & Transporte Público \\
\hline 15 minutos o menos & $67 \%$ & $56 \%$ \\
\hline 16 a 30 minutos & $26 \%$ & $33 \%$ \\
\hline 31 a 45 minutos & $4 \%$ & $7 \%$ \\
\hline Más de 45 minutos & $3 \%$ & $4 \%$ \\
\hline
\end{tabular}

\section{Distancia caminada para acceder al transporte público}

El indicador de "distancia caminada para acceder al transporte público" expone una de las variables más importantes que inciden en la elección modal del usuario del sistema transporte público, ya que la extensión de la caminata está relacionada con diversos aspectos, tales como: la accesibilidad de atravesar el espacio físico que presenta para el usuario, los incrementales tiempos de viaje o transbordo, la seguridad a lo largo del recorrido caminado, $\mathrm{u}$ otras particularidades para determinados grupos (por ejemplo, las condiciones de la accesibilidad al sistema de transporte público va a variar según si los usuarios presentan movilidad reducida, si existen condiciones climáticas adversas, según la hora del día, si el usuario se transporta con bolsos pesados o materiales de estudio, entre otros).

Este indicador es uno de los más significativos del nivel de accesibilidad al transporte público de las personas, de acuerdo con su localización y en relación con el sistema de transporte de la ciudad.

Los resultados generales de distancia caminada son contundentes, reflejando la diferencia de caminata de acuerdo con los grupos de edad. El mismo análisis se realizó según los cortes territoriales, entendiendo que se podían esperar diferentes resultados de acuerdo con la compacidad de la urbanización y distancias a servicios de transporte, como se observa en los cuadros 4 y 5.

Cuadro 4. Cuadras caminadas para viajes en transporte público, según grupo de NNA. Fuente: Elaboración propia, en base a Encuesta de Movilidad en el Área Metropolitana de Buenos Aires, ENMODO, 2009-2010.

\begin{tabular}{|l|c|c|c|}
\hline Cuadras caminadas & NNA 3 a 5 años & NNA 6 a 12 años & NNA 13 a 17 años \\
\hline No camina & $6,3 \%$ & $6,5 \%$ & $5,3 \%$ \\
\hline Una a dos & $29,5 \%$ & $29,5 \%$ & $22,5 \%$ \\
\hline Tres a cinco & $38,5 \%$ & $38,5 \%$ & $38,7 \%$ \\
\hline Seis a nueve & $19,7 \%$ & $18,2 \%$ & $24,0 \%$ \\
\hline Diez o más & $6,0 \%$ & $7,3 \%$ & $9,5 \%$ \\
\hline
\end{tabular}

Cuadro 5. Cuadras caminadas para viajes en transporte público, total NNA por localización. Fuente: Elaboración propia, en base a Encuesta de Movilidad en el Área Metropolitana de Buenos Aires, ENMODO, 2009-2010.

\begin{tabular}{|l|c|c|c|}
\hline Cuadras caminadas & CABA & Corona 1 & Corona 2 \\
\hline No camina & $4,9 \%$ & $7,9 \%$ & $5,7 \%$ \\
\hline Una a dos & $36,0 \%$ & $28,7 \%$ & $21,4 \%$ \\
\hline Tres a cinco & $41,3 \%$ & $36,5 \%$ & $39,5 \%$ \\
\hline Seis a nueve & $12,5 \%$ & $22,2 \%$ & $21,8 \%$ \\
\hline Diez o más & $5,3 \%$ & $4,7 \%$ & $11,6 \%$ \\
\hline
\end{tabular}




\section{Tiempos de espera del transporte público}

Los tiempos de espera están asociados principalmente a la estancia en las paradas y/o estaciones de transporte público. Depende de la frecuencia de los servicios, es decir del tiempo que existe entre un servicio y otro, y de la confiabilidad de su regularidad. Este tiempo va a depender, generalmente, de al menos tres factores primordiales que afectan a la frecuencia: a) el tipo de servicio de transporte que se espera (colectivo, ferrocarril, subte); b) la localización de la parada o estación en la que se espera el servicio en relación a la traza del mismo (no es lo mismo la cabecera - inicio - del servicio, que una parada o estación en la mitad del recorrido, por ejemplo) y c) el barrio o localidad en el que se encuentre la parada o estación (no es lo mismo el centro de la ciudad que la periferia).

En el cuadro 4 se presentan los resultados para todos los NNA por recorte territorial. Los resultados desagregados por grupo de NNA no fueron significativos estadísticamente y por lo tanto se descartaron, probablemente porque "los tiempos de espera" son resultado del tipo de servicio más que de las características de los NNA.

De esta manera se pudieron obtener resultados significativamente diferentes según los recortes territoriales definidos, como puede observarse en el cuadro 6:

Cuadro 6. Tiempos de espera en el transporte público, según zona geográfica. Fuente: Elaboración propia, en base a Encuesta de Movilidad en el Área Metropolitana de Buenos Aires, ENMODO, 2009-2010.

\begin{tabular}{|l|c|c|c|}
\hline Tiempos de espera & CABA & CORONA 1 & CORONA 2 \\
\hline 10 minutos o menos & $82,20 \%$ & $76,60 \%$ & $66,80 \%$ \\
\hline 11 a 20 minutos & $14,10 \%$ & $18,20 \%$ & $24,50 \%$ \\
\hline Más de 20 minutos & $3,70 \%$ & $5,20 \%$ & $8,60 \%$ \\
\hline
\end{tabular}

Del cuadro precedente pueden diferenciarse los tiempos de espera según la ubicación geográfica de los usuarios: a medida que estos se encuentran más alejados del centro de la ciudad, los tiempos de espera al transporte público son mayores. Por otra parte, se destaca que los tiempos de espera en CABA son considerablemente menores que en Corona 1 y 2: en el caso de diez minutos o menos, se obtiene la mayor proporción - con más del 82\%-, reduciéndose al 66,80\% cuando estos tiempos se dan en Corona 2. Esto se debe a que la frecuencia o regularidad de los servicios públicos es menor a medida que nos extendemos en el territorio hacia la periferia del AMBA, penalizando en los usuarios el tiempo de espera al arribar el servicio público, mayormente, cuando refiere a servicios de colectivos urbanos.

\section{Viajes escolares de adulto acompañante}

Estos datos solo pudieron analizarse para el conjunto del AMBA, no pudiendo desagregarse en recortes territoriales menores por la baja representatividad de la muestra en los municipios. No obstante, es interesante realizar la segmentación puesto que este indicador demuestra como son los viajes de los adultos que acompañan a los NNA, sobre todo de aquellos con diferentes niveles socioeconómicos, diferentes discapacidades-capacidades de movilidad, diferentes niveles de accesibilidad al sistema de transporte, diferente género, etc.

Como resultado, se obtiene que los hogares con nivel socioeconómico (NSE) bajo realizan más viajes en calidad de acompañante a un NNA a centro educativo (65\%) que los hogares con NSE alto (52\%). Además, se identificó que en el AMBA son principalmente mujeres quienes cumplen con la función de acompañar a los NNA a un centro educativo: $74,4 \%$ de los viajes como acompañantes son realizados por mujeres, mientras que los hombres lo hacen en el 25,6\% de los viajes. 


\section{Principales hallazgos y recomendaciones}

La ENMODO es una encuesta domiciliaria muy extensa y compleja en términos de la gran cantidad de información que recolectó y el despliegue de recursos en campo y escritorio que precisó, tanto para realizar el trabajo de recolección de la muestra, como de supervisión, validación y sistematización de resultados en un recorte territorial tan amplio como es el AMBA.

La encuesta fue realizada con el objetivo de comprender en forma agregada o estratégica las principales características de la movilidad del área metropolitana, como identificar grandes atractores y generadores de viajes, determinar la utilización de la red de movilidad (principal y no local), entre otros. En este sentido, la muestra no alcanzo el 1\% de la población del AMBA, resultando en procesamientos limitados a niveles territoriales menores, como lo son los municipios o las comunas.

Por lo tanto, al momento de realizar estudios específicos de movilidad como por ejemplo el análisis de la accesibilidad hacia centros educativos del grupo poblacional de los NNA que realizaron viajes un día hábil promedio en el año 2010, ha resultado un análisis complejo, en el cual se tuvo que analizar en detalle cada una de las variables o indicadores a ser tomados, como así también entender el tamaño de la muestra ya no en general, sino en el particular de cada indicador seleccionado.

Realizar esta segmentación de variables permitió evidenciar patrones diferenciados según el territorio y el grupo social: se ha podido constatar en detalle como son las características de accesibilidad y movilidad para la población de estudiantes de entre 3 y 17 años.

A su vez, este análisis permitió demostrar que más del $50 \%$ de los viajes por motivos educativos de los NNA se realiza en modos no motorizados (a pie o en bicicleta) y que más de la mitad de aquellos viajes realizados a pie son de una distancia promedio de tres a nueve cuadras.

En cuanto al análisis de los grupos de NNA, el grupo de primera infancia ( 3 a 5 años) es aquel que más se desplaza en modos no motorizados para acceder a los centros educativos, lo cual sea probablemente por la utilización de centros educativos de proximidad o barriales, cercanos al hogar.

En este sentido, y para completar un análisis de accesibilidad, se hace necesario evaluar como son las condiciones de "caminabilidad" en el AMBA, desde los hogares hacia los centros educativos, teniendo en cuenta el estado de las aceras y de las calles, la cantidad de obstáculos o barreras que encuentran los NNA al momento de realizar los viajes a pie.

Por otro lado, se ha observado que solo el 4,3\% de los viajes de los NNA de 3 a 5 años realizan el trayecto hacia el establecimiento educativo en bicicleta, con lo cual este indicador resulta primordial para decidir sobre política pública al respecto, ya que conociendo las rutas por las que se accede a los centros educativos se pueden realizar propuestas de creación de senderos de tipo "bicisendas" o "ciclovías", para incentivar este modo, aportar mayor seguridad vial y mejor accesibilidad en estos viajes.

Por su parte, el segundo grupo de NNA de 6 a 12 años, refleja condiciones de movilidad con mayor proporción de utilización del transporte público respecto de los de primera infancia, incrementándose aún más cuando se observan los datos de los NNA de 13 a 17 años. 
$\mathrm{Al}$ analizar los resultados de los tiempos de espera al transporte público según zona geográfica, estos son contundentes: en CABA el 82,2\% de los NNA espera 10 minutos o menos, mientras que en la Corona 2 este porcentaje baja a 66,8\%. De la misma manera, aquellos NNA que esperan más de 20 minutos, son solo un 3,7\% en CABA respecto del $8,6 \%$ de Corona 2 , con lo cual se concluye que a medida que nos alejamos del centro del AMBA, los tiempos de espera al transporte público son mayores, lo cual hace reflexionar sobre cómo es la calidad de la provisión del servicio en estas zonas más alejadas.

Es de destacar que el estudio ha querido abordar el análisis de movilidad del grupo poblacional como el de las mujeres en su rol de "acompañante" a los NNA en sus distintas actividades; no obstante, el mismo no pudo llevarse a cabo puesto que no existe representatividad muestral en dicho indicador para los tres grupos de NNA y para las distintas áreas geográficas de análisis.

En este sentido, el uso de este tipo de encuestas que muestralmente fueron pensadas con fines de política estratégica, no son óptimas para realizar análisis desagregados, pero su uso permite un entendimiento global de la movilidad que luego puede complementarse con otras herramientas y fuentes de datos que permitan una mayor desagregación de las variables en el territorio, para abordar a conclusiones más específicas. 


\section{Q Bibliografía}

» Abba, A. F. (2011). Revelaciones de los datos provisionales del Censo 2010: ¿Una nueva realidad de la Buenos Aires Metropolitana? Observatorio Urbano Local Buenos Aires Metropolitana.

» Anapolsky, S., y Pereyra, L. (2012). Desafíos de la gestión y la planificación del transporte urbano y la movilidad de las ciudades argentinas. Revista Transporte y Territorio, 7, 57-75.

"Galeota, C. y Neri, N. (2020). La movilidad y las condiciones de accesibilidad a centros educativos en el Área Metropolitana de Buenos Aires. En La agenda emergente de las políticas sociales. Movilidad urbana, cuidado y violencias de género. Pautassi, L. (coomp.). Buenos Aires: Biblos.

"Capelli, L. (2015). Analysing the relation between public transport provision and social exclusion in the metropolitan area of Buenos Aires. Leeds: University of Leeds.

"ENMODO (2010). Resultados de la Encuesta Origen Destino 2009-2010. Disponible en https://www.argentina.gob.ar/transporte/dgppse/publicaciones/ encuestas

》 Gutiérrez, A. (2010). Movilidad, transporte y acceso: una renovación aplicada al ordenamiento territorial. Scripta Nova: revista electrónica de geografía y ciencias sociales, 86.

" Keeling, D. J. (2008). Latin America's transportation conundrum. Journal of Latin American Geography, 7, 133-154.

» Titheridge, H. (2006). Social exclusion and transport policy. University College London.

»Torres, H. A. (2004). Procesos recientes de fragmentación socioespacial en Buenos Aires: la suburbanización de las élites. Revista digital "Mundo Urbano" de la Universidad Nacional de Quilmes, 3, mayo de 2004.

\section{Carla Galeota / cgaleba@gmail.com}

Arquitecta (UBA), Magíster of Science en Planificación del Transporte (Universidad de Leeds). Docente e Investigadora (UNSAM. Carrera de Tecnicatura y Licenciatura en Transporte). Docente de Posgrado (UNSAM).

\section{Natalia Verónica Neri / nataliavneri@gmail.com}

Licenciada en Geografía (UBA). Subgerente de Transporte Urbano de Pasajeros (CNRT). Docente (UNTREF. Carreras de Licenciaturas en SIG y Geografía). Docente e Investigadora del Instituto de Transporte (UNSAM).

\section{Lucila Capelli / capelli.lucila@gmail.com}

Licenciada en Ciencias Políticas (UBA). Magíster of Science en Planificación del Transporte y Medio Ambiente (Universidad de Leeds). Subsecretaria de Planificación de la Movilidad (GCABA). Docente e Investigadora del Instituto de Transporte (UNSAM). 